\title{
ON THE EXISTENCE AND CHARACTERIZATION OF BEST NONLINEAR TCHEBYCHEFF APPROXIMATIONS
}

BY

JOHN R. RICE

1. Introduction. This paper is concerned with the following approximation problem:

Tchebycheff approximation problem. Let $f(x)$ be continuous on $[0,1]$ and let $F(A, x)$ be a continuous approximating function depending on $n$ parameters, $A=\left(a_{1}, a_{2}, \cdots, a_{n}\right)$. Denote by $P$ the domain of the parameters. Given $f(x)$ determine $A^{*} \in P$ such that

$$
\max _{x \in[0.1]}\left|F\left(A^{*}, x\right)-f(x)\right| \leqq \max _{x \in[0,1]}|F(A, x)-f(x)|
$$

for all $A \in P$. Such an $F\left(A^{*}, x\right)$ is a best approximation to $f(x)$.

Relative to this problem there are three principal statements to be investigated. They are

StATEMENT A. $f(x)$ possesses a best approximation.

Statement B. Best approximations are characterized as those $F\left(A^{*}, x\right)$ for which $F\left(A^{*}, x\right)-f(x)$ alternates at least $n$ times on $[0,1]$.

STATEMENT C. The best approximation is unique.

Statement B gives the usual characteristic property of best Tchebycheff approximations. The function $F\left(A^{*}, x\right)-f(x)$ is said to alternate $n$ times if there are $n+1$ points

$$
0 \leqq x_{1}<x_{2}<\cdots<x_{n+1} \leqq 1
$$

such that

$$
F\left(A^{*}, x_{j}\right)-f\left(x_{j}\right)=-\left[F\left(A^{*}, x_{j+1}\right)-f\left(x_{j+1}\right)\right]= \pm \max \left|F\left(A^{*}, x\right)-f(x)\right| .
$$

All maxima in this paper are taken over $x \in[0,1]$ unless otherwise stated.

The usual effort on a problem of this type is to be given a particular $F(A, x)$ and then to attempt to establish one or more of Statements A, B and C. The effort presented in this paper is one of a more general and partially converse nature. This study concerns the following question: What conditions on $F(A, x)$ are both necessary and sufficient for a certain combination of Statements A, $\mathrm{B}$ and $\mathrm{C}$ to be valid for all continuous functions?

Received by the editors February 7, 1962 and, in revised form, May 22, 1962, August 13, 1962, November 30, 1962 and January 25, 1963. 
Such a question was first posed and answered by Haar [2] where, for linear approximating functions

$$
F(A, x)=\sum_{i=1}^{n} a_{i} \phi_{i}(x),
$$

he asked what are necessary and sufficient conditions for Statement $\mathbf{C}$ to be valid for all continuous functions. More recently Rice [4] has posed and answered the question (for linear approximating functions) for Statement A and for Statements A and B. Also Rice [5] has answered this question for Statement B and for a continuous nonlinear approximating function.

In this paper conditions on a nonlinear approximating function $F(A, x)$ are found which are necessary and sufficient for both Statements A and B to be valid for all continuous functions.

The conditions found to be equivalent to Statements A and B are closure under pointwise convergence (Definition 4) and local unisolvence (Definition 3).

In the final section it is noted that if Statement $B$ is valid for all continuous functions, then so is Statement $C$. This implies that the condition found here for Statements A and B are also the conditions for Statements A, B and C.

2. The theorem and proof. In order to describe the properties of $F$ that characterize Statements A and B, and in order to facilitate the discussion, the following definitions are made.

Definition 1. F has Property $\mathrm{Z}$ if $A^{*} \neq A$ implies that

$$
F\left(A^{*}, x\right)-F(A, x)
$$

has at most $n-1$ zeros in $[0,1]$.

Definition 2. $F$ is said to be locally solvent if given $0 \leqq x_{1}<x_{2}<\cdots<x_{n} \leqq 1$, $A^{*} \in P$ and $\varepsilon>0$ then there is a $\delta\left(A^{*}, \varepsilon, x_{1}, x_{2}, \cdots, x_{n}\right)>0$ such that

$$
\left|F\left(A^{*}, x_{j}\right)-y_{j}\right|<\delta
$$

implies the existence of a solution $A \in P$ to

$$
F\left(A, x_{j}\right)=y_{j}
$$

with $\max \left|F(A, x)-F\left(A^{*}, x\right)\right|<\varepsilon$.

DEFINITION 3. $F$ is said to be locally unisolvent if $F$ is locally solvent and has Property $\mathrm{Z}$.

The final property is closely related to unisolvence [3]. In both cases Property $Z$ is present. For $F$ unisolvent one is assured of solving (2.1) for any set of values $\left\{y_{j}\right\}$, the present definition only assures a solution of (2.1) if the points $\left\{\left(x_{j}, y_{j}\right) \mid j=1,2, \cdots, n\right\}$ lie in a neighbohood of some curve $F\left(A^{*}, x\right)$.

The next definition describes a property to be associated with Statement A.

Definition 4. $F$ is said to be closed if $P$ is arcwise connected and if $F$ is closed under pointwise limits, i.e., 


$$
\operatorname{Lim}_{k \rightarrow \infty} F\left(A_{k}, x\right)=G(x), \quad x \in[0,1], \quad\left|F\left(A_{k}, x\right)\right| \leqq M
$$

implies there is an $A_{0} \in P$ such that

$$
F\left(A_{0}, x\right) \equiv G(x) .
$$

At this point it is appropriate to make a remark on the topology of the parameter space $P$. Since $F(A, x)$ depends on $n$ parameters, one naturally associates the parameters $A$ with a point in Euclidean $n$-space $E_{n}$. However the topology of $E_{n}$ may not be suitable for $P$ and indeed it may be extremely difficult to imbed $P$ in $E_{n}$ in such a way that the $E_{n}$ topology has any meaning at all for $F$. Thus $P$ is considered to be an abstract space with its topology derived from the uniform norm on the set of functions $\{F(A, x)\}$. In this way the statement

$$
\operatorname{Lim}_{k \rightarrow \infty} A_{k}=A^{*}
$$

is defined to be equivalent to

$$
\operatorname{Lim}_{k \rightarrow \infty} \max \left|F\left(A_{k}, x\right)-F\left(A^{*}, x\right)\right|=0 .
$$

If $F$ is locally solvent then one may show that pointwise closure (2.2) becomes uniform closure (2.5).

The above definitions were originally made in [5]. The definition of closure here has been made slightly less restrictive. For the application of the results of [5] in this paper, the definitions are equivalent.

The main thorem of this paper answers the question posed in the introduction, namely, what does the validity of Statements A and B for every continuous $f(x)$ imply about the properties of the approximating function $F(A, x)$, and vice versa.

THEOREM 1. Statements A and B are valid for every continuous function if and only if $F$ is closed and locally unisolvent.

A portion of the proof of this theorem is found in [5]. There are two points which remain to be established. The first and simplest is that the closure of $F$ (along with Property $\mathrm{Z}$ ) implies the existence of best approximations for every continuous function. The second is that the validity of Statements A and B imply that $F$ is closed.

Lemma 1. If $F$ is closed and has Property $\mathrm{Z}$ then Statement $\mathrm{A}$ is valid for every continuous function.

Proof. Let $f(x)$ be a given function continuous on $[0,1]$ and $F\left(A^{\prime}, x\right)$ an approximating function. Denote by $P^{\prime}$ the parameters

$$
P^{\prime}=\left\{A|\max | F(A, x)-f(x)|\leqq \max | F\left(A^{\prime}, x\right)-f(x) \mid\right\} .
$$


This set is not empty since it contains $A^{\prime}$. Furthermore it is a bounded set, i.e., there exists an $M<\infty$ such that $|F(A, x)| \leqq M$ for $A \in P^{\prime}$.

There is a sequence $\left\{A_{k}\right\}$ in $P^{\prime}$ such that

$$
\operatorname{Lim}_{k \rightarrow \infty} \max \left|F\left(A_{k}, x\right)-f(x)\right|=\inf _{A \in P} \max |F(A, x)-f(x)| .
$$

It is known [5, Theorem 2] that Property $Z$ implies the existence of a pointwise convergent subsequence of every infinite sequence in $P^{\prime}$. If $P^{\prime}$ contains only a finite number of parameter sets then Statement A is clearly valid for $f(x)$. Thus the sequence $\left\{F\left(A_{k}, x\right)\right\}$ contains a pointwise convergent subsequence and, by the hypothesis of closure, this subsequence possesses a limit $F\left(A_{0}, x\right)$ which is a best approximation to $f(x)$.

In order to establish the second point, one would like to construct for $G(x)$ a continuous function $f(x)$ such that $f(x)-G(x)$ alternates $n$ times at $n+1$ specified points in $[0,1]$. This would imply (after some arguments) that $G(x)$ is a best approximation to $f(x)$ and hence (by Statement A) that $G(x) \equiv F\left(A_{0}, x\right)$. However it is not possible at this point to construct such an $f(x)$ since $G(x)$ is an unknown and possibly highly discontinuous function. This difficulty is circumvented in Lemma 2 where two functions associated with $G(x)$ are introduced for which one may construct the required continuous function $f(x)$. These two functions are

$$
\begin{aligned}
& G^{+}(x)=\max [G(x), \quad \underset{|x-y| \rightarrow 0}{\operatorname{Limsup}} G(y)], \\
& G^{-}(x)=\min [G(x), \underset{|x-y| \rightarrow 0}{\operatorname{Liminf}} G(y)] .
\end{aligned}
$$

Since $G(x)$ is a bounded function, both of these functions are well defined.

Lemma 2. Given $G(x)$ bounded on $[0,1], M>0, \delta_{0}>0$ and $x_{0} \in[0,1]$ there exists a continuous function $f(x)$ such that

$$
f(x)-G^{+}(x)
$$

has a minimum $-M$ at $x_{0}$ in the interval $\left|x-x_{0}\right| \leqq \delta_{0}$. Further

$$
f\left(x_{0} \pm \delta_{0}\right)-G^{+}\left(x_{0} \pm \delta_{0}\right)=0 \text {. }
$$

Proof. Set

$$
\omega^{+}(\delta)=\sup \left[G^{+}(y)-G^{+}\left(x_{0}\right)\right], \quad 0<\left|x_{0}-y\right|<\delta .
$$

This is an "upper modulus of continuity" of $G^{+}(x)$ at $x_{0}$. It is also the uppersemicontinuous function $u(x)$ (upper boundary function) described in [6, Chapter 7]. If

$$
G\left(x_{0}\right)=G^{+}\left(x_{0}\right)>\underset{\left|x_{0}-y\right| \rightarrow 0}{\operatorname{Lim} \sup _{\mid}} G(x)
$$


then clearly

$$
\operatorname{Lim}_{\delta \rightarrow 0} \omega^{+}(\delta)<0 .
$$

When (2.9) does not hold then we have the following assertion: If

then

$$
G\left(x_{0}\right) \leqq G^{+}\left(x_{0}\right)
$$

$$
\operatorname{Lim}_{\delta \rightarrow 0} \omega^{+}(\delta)=0 .
$$

The basic reason that this assertion is true is that $G^{+}(x)$ itself is upper-semicontinuous Assume (2.10) to be false, then there is an $\varepsilon>0$ and a sequence $\left\{x_{i} \mid i=1,2, \cdots\right\}$ tending to $x_{0}$ such that

$$
G^{+}\left(x_{0}\right)<G^{+}\left(x_{i}\right)-\varepsilon, \quad i=1,2, \cdots .
$$

This contradicts the fact

$$
G^{+}\left(x_{0}\right) \geqq \underset{\left|x_{0}-y\right| \rightarrow 0}{\operatorname{Lim} \sup } G^{+}(y)
$$

which may be established by a straightforward argument.

A construction is now given to establish the following

AsSERTION. There exists a continuous function $\omega(\delta)$ such that for $0 \leqq \delta \leqq \delta_{0}$

$$
\omega^{+}(\delta) \leqq \omega(\delta)
$$

and if (2.10) holds then $\omega(0)=0$.

Note that $\omega^{+}(\delta)$ is a monotonic nondecreasing function. Define

$$
\begin{aligned}
& \omega(\delta)=\frac{1}{\delta} \int_{\delta}^{2 \delta} \omega^{+}(x) d x, \quad \delta>0, \\
& \omega(0)=\operatorname{Limsup}_{\left|x_{0}-y\right| \rightarrow 0} G(y)-G^{+}\left(x_{0}\right) .
\end{aligned}
$$

It follows immediately from the mean value theorem that

$$
\omega^{+}(\delta) \leqq \omega(\delta) \leqq \omega^{+}(2 \delta), \quad \delta>0 .
$$

It is clear that $\omega(\delta)$ is a continuous function and further if (2.10) holds then $\omega(0)=0$.

The function $f(x)$ required in this proof is now constructed. If $\omega\left(\delta_{0}\right) \leqq 0$ set

$$
f(x)=G^{+}\left(x_{0}\right)-M+2\left|x-x_{0}\right| \text {. }
$$

Then if $\left|x-x_{0}\right| \leqq \delta_{0}$ one has

$$
f(x)-G^{+}(x)=G^{+}\left(x_{0}\right)-G^{+}(x)-M+2\left|x-x_{0}\right| \geqq-M+2\left|x-x_{0}\right| .
$$

If $\omega\left(\delta_{0}\right)>0$ set $\omega^{\prime}(\delta)=\max [\omega(\delta), 0]$ and

$$
f(x)=G^{+}\left(x_{0}\right)-M+2 \omega^{\prime}\left(\left|x-x_{0}\right|\right) \text {. }
$$


Then

$$
\begin{aligned}
f(x)-G^{+}(x) & =G^{+}\left(x_{0}\right)-G^{+}(x)-M+2 \omega^{\prime}\left(\left|x-x_{0}\right|\right) \\
& \geqq-M+2 \omega^{\prime}\left(\left|x-x_{0}\right|\right)-\omega^{+}\left(\left|x-x_{0}\right|\right) \\
& \geqq-M+\omega^{\prime}\left(\left|x-x_{0}\right|\right) \geqq-M .
\end{aligned}
$$

This construction gives an $f(x)$ satisfying the minimum requirement of the lemma. The construction may be easily modified so that

$$
f\left(x_{0} \pm \delta_{0}\right)=G^{+}\left(x_{0} \pm \delta_{0}\right) \text {. }
$$

It is clear that the same type of construction is applicable to the

Corollary. Given $G(x)$ bounded on $[0,1], M>0, \delta_{0}>0$ and $x_{0} \in[0,1]$ then there exists a continuous function $f(x)$ such that

$$
f(x)-G^{-}(x)
$$

has a local maximum of $M$ at $x_{0}$ in the interval $\left|x-x_{0}\right| \leqq \delta_{0}$. Further

$$
f\left(x_{0} \pm \delta_{0}\right)-G^{-}\left(x_{0} \pm \delta_{0}\right)=0 .
$$

The next lemma is required to establish the second point of the proof of Theorem 1. This lemma is a restatement of some results in [5], particularly Lemma 4 and Theorem 1.

LEMMA 3. Assume Statement B is valid for every continuous function. If

$$
\max _{x_{i}}\left|F\left(A, x_{i}\right)-f\left(x_{i}\right)\right| \leqq 2 M, \quad i=1,2, \cdots, n+1
$$

then either (i) $F(A, x)-f(x)$ alternates $n$ times on $\left\{x_{i}\right\}$ with deviation $2 M$ or (ii) there is an $A_{0} \in P$ such that

$$
\left|F\left(A_{0}, x_{i}\right)-f\left(x_{i}\right)\right|<2 M, \quad i=1,2, \cdots, n+1 .
$$

The next lemma establishes the second point required for the proof of Theorem 1.

LEMMA 4. If Statements $\mathrm{A}$ and $\mathrm{B}$ are valid for every continuous function then $F$ is closed.

Proof. Assume that

$$
\operatorname{Lim}_{k \rightarrow \infty} F\left(A_{k}, x\right)=G(x), \quad x \in[0,1]
$$

with

$$
\left|F\left(A_{k}, x\right)\right| \leqq M
$$

Let $n+1$ points be given

$$
0 \leqq x_{1}<x_{2}<\cdots<x_{n+1} \leqq 1
$$

and set $\delta_{0}=\frac{1}{4} \min \left|x_{j}-x_{j+1}\right|$. 
By Lemma 2 a continuous function $f_{1}(x)$ may be defined by (2.12) so that

$$
\begin{array}{ll}
f_{1}\left(x_{j}\right)-G^{-}\left(x_{j}\right)=+2 M, & j=1,3,5, \cdots, \\
f_{1}\left(x_{j}\right)-G^{+}\left(x_{j}\right)=-2 M, & j=2,4, \cdots
\end{array}
$$

and these points are local extrema of $f_{1}(x)-G^{+}(x)$ and $f_{1}(x)-G^{-}(x)$ in $\left[x_{j}-\delta_{0}, x_{j}+\delta_{0}\right]$. Further, the definition of $f_{1}(x)$ may be extended to the remainder of $[0,1]$ so that

$$
\left|f_{1}(x)-G(x)\right| \leqq M, \quad\left|x-x_{j}\right|>\delta_{0} .
$$

Since $f_{1}(x)$ is continuous, the assumption that Statement $\mathrm{A}$ is valid implies that $f_{1}(x)$ possesses a best approximation $F\left(A^{1}, x\right)$. The assumption that Statement $B$ is valid implies [5, Lemma 3] that $F$ has Property $Z$. This fact is used essentially in the remainder of the proof.

The following assertion is now established:

ASSERTION 1.

$$
\max \left|f_{1}(x)-F\left(A^{1}, x\right)\right| \leqq 2 M .
$$

Since Statement $\mathrm{B}$ is valid there are $n+1$ points $\left\{y_{j} \mid y_{j}<y_{j+1}\right\}$ such that

$$
F\left(A^{1}, y_{j}\right)-f_{1}\left(y_{j}\right)=(-1)^{j} K,
$$

where $K= \pm \max \left|f_{1}(x)-F\left(A^{1}, x\right)\right|$. If $|K|>2 M$ then since $\left|G\left(y_{j}\right)-f_{1}\left(y_{j}\right)\right|$ $\leqq 2 M<|K|$ one has

$$
\left[F\left(A^{1}, y_{j}\right)-G\left(y_{j}\right)\right](-1)^{j} \operatorname{sgn}[K] \geqq|K|-2 M>0 .
$$

For $k$ sufficiently large one has

$$
\left|F\left(A_{k}, y_{j}\right)-G\left(y_{j}\right)\right|<\frac{1}{2}(|K|-2 M)
$$

and hence

$$
\operatorname{sgn}\left[F\left(A^{1}, y_{j}\right)-F\left(A_{k}, y_{j}\right)\right]=(-1)^{j} \operatorname{sgn}[K] .
$$

This implies that $F$ does not have Property $\mathrm{Z}$ which contradicts the assumption that Statement $B$ is valid for all continuous functions. This establishes the assertion (2.16).

We now establish

ASSERTION 2.

$$
\left|F\left(A^{1}, x_{j}\right)-f_{1}\left(x_{j}\right)\right|=2 M, \quad j=1,2, \cdots, n+1
$$

It follows from the first assertion that

$$
\left|F\left(A^{1}, x_{j}\right)-f_{1}\left(x_{j}\right)\right| \leqq 2 M, \quad j=1,2, \cdots, n+1 .
$$

Lemma 3 implies that either (2.17) follows from (2.18) or there is an $A_{0} \in P$ such that 


$$
\left|F\left(A_{0}, x_{j}\right)-f_{1}\left(x_{j}\right)\right|<2 M, \quad j=1,2, \cdots, n+1 .
$$

It is now shown that the alternative (2.19) leads to a contradiction.

Set

$$
\varepsilon=\min _{j}\left[2 M-\left|F\left(A_{0}, x_{j}\right)-f_{1}\left(x_{j}\right)\right|\right]>0 .
$$

There is an $\eta>0$ such that $\left|x-x_{j}\right|<\eta$ implies

$$
\left|F\left(A_{0}, x_{j}\right)-F\left(A_{0}, x\right)\right|<\frac{\varepsilon}{3}, \quad j=1,2, \cdots, n+1 .
$$

Further, there exists a $y_{j}$ with $\left|y_{j}-x_{j}\right|<\eta$ so

$$
\left|G\left(y_{j}\right)-G^{ \pm}\left(x_{j}\right)\right|<\frac{\varepsilon}{3}, \quad j=1,2, \cdots, n+1 .
$$

One may choose $k$ so large that

$$
\left|G\left(y_{j}\right)-F\left(A_{k}, y_{j}\right)\right|<\frac{\varepsilon}{3}, \quad j=1,2, \cdots, n+1 .
$$

With alternative (2.19) it follows from these estimates that

$$
\begin{array}{ll}
F\left(A_{0}, y_{j}\right)>F\left(A_{k}, y_{j}\right), & j \text { odd, } \\
F\left(A_{0}, y_{j}\right)<F\left(A_{k}, y_{j}\right), & j \text { even. }
\end{array}
$$

This implies that $F\left(A_{0}, x\right)-F\left(A_{k}, x\right)$ has $n$ zeros which is impossible. This establishes the assertion.

These two assertions imply that

$$
\begin{array}{ll}
F\left(A^{1}, x_{j}\right)=G^{-}\left(x_{j}\right), & j \text { odd, } \\
F\left(A^{1}, x_{j}\right)=G^{+}\left(x_{j}\right), & j \text { even. }
\end{array}
$$

A similar construction of a continuous function $f_{2}(x)$ and an analysis of a best approximation $F\left(A^{2}, x\right)$ to it leads to

$$
\begin{array}{ll}
F\left(A^{2}, x_{j}\right)=G^{+}\left(x_{j}\right), & j \text { odd, } \\
F\left(A^{2}, x_{j}\right)=G^{-}\left(x_{j}\right), & j \text { even. }
\end{array}
$$

It follows from (2.20) and (2.21) that

$$
\left[F\left(A^{2}, x_{j}\right)-F\left(A^{1}, x_{j}\right)\right](-1)^{j+1} \geqq 0, \quad j=1,2, \cdots, n+1 .
$$

Since $F$ must have Property $Z$, this implies that

$$
F\left(A^{2}, x_{j}\right) \equiv F\left(A^{1}, x_{j}\right) .
$$

Since

it follows that

$$
G^{+}(x) \geqq G(x) \geqq G^{-}(x)
$$




$$
G\left(x_{j}\right)=F\left(A^{1}, x_{j}\right), \quad j=1,2, \cdots, n+1 .
$$

One may fix $n$ distinct points and let the $(n+1)$ st point be variable. Then $(2.24)$ is valid with $A^{1}$ replaced by a new parameter set $A_{0}$. However, on the $n$ fixed points one has $F\left(A^{1}, x_{j}\right)=F\left(A_{0}, x_{j}\right), j=1,2, \cdots, n$ which implies that $F\left(A^{1}, x\right)=F\left(A_{0}, x\right)$. Thus one has for any $x$,

$$
G(x)=F\left(A^{1}, x\right) .
$$

This is the approximating function required in this lemma and concludes the proof.

Proof of Theorem 1. There are two implications to be established: (i) local unisolvence and closure imply Statements A and B and (ii) Statements A and B imply local unisolvence and closure.

For the first implication we have shown (Lemma 1) that local unisolvence (which includes Property Z) and closure imply Statement A. It is known [5, Theorem 3] that local unisolvence and closure imply Statement B.

For the second implication it is known [5, Lemma 3] that Statement B implies Property Z. We have shown (Lemma 4) that Statements A and B imply closure. It is known [5, Theorem 3] that Statement B and closure (and hence Statements A and B) imply local unisolvence. This concludes the proof.

3. Example. For linear approximating functions, it is known [4] that the classical $\left({ }^{1}\right)$ approximating functions are the only ones for which Statements $\mathrm{A}, \mathrm{B}$ and $\mathrm{C}$ are all valid for all continuous functions. The simplest and most "classical" nonlinear approximating functions are the unisolvent functions [3]. It is known that Statements A, B and C are valid for these approximating functions. One might conjecture then that these are the only approximating functions for which Statements A, B and C are all valid. That this is not true is seen by the simple example

$$
F(A, x)=\frac{a}{1+a x}, \quad-1<a<+1, \quad-1 \leqq x \leqq+1 .
$$

One may easily verify that the three Statements A, B and C are valid for all continuous functions. The range of this approximating function is shown in Figure 1.

4. Remark on Haar's problem and uniqueness. The following results are known [5, Lemma 3, and classical]:

1. If Statement $B$ is valid for every continuous function then $F$ has Property $Z$.

2. If $F$ has Property $Z$ then Statement $C$ is valid for every continuous function. These results have the obvious corollary, which has not been explicitly stated previously.

(1) The classical linear approximating functions are $F(A, x)=\sum_{i=1}^{n} a_{i} \varphi_{i}(x)$, where $P=E_{n}$ and $\left\{\varphi_{i}(x)\right\}$ is a Tchebycheff set, i.e., $F$ has Property $Z$. 


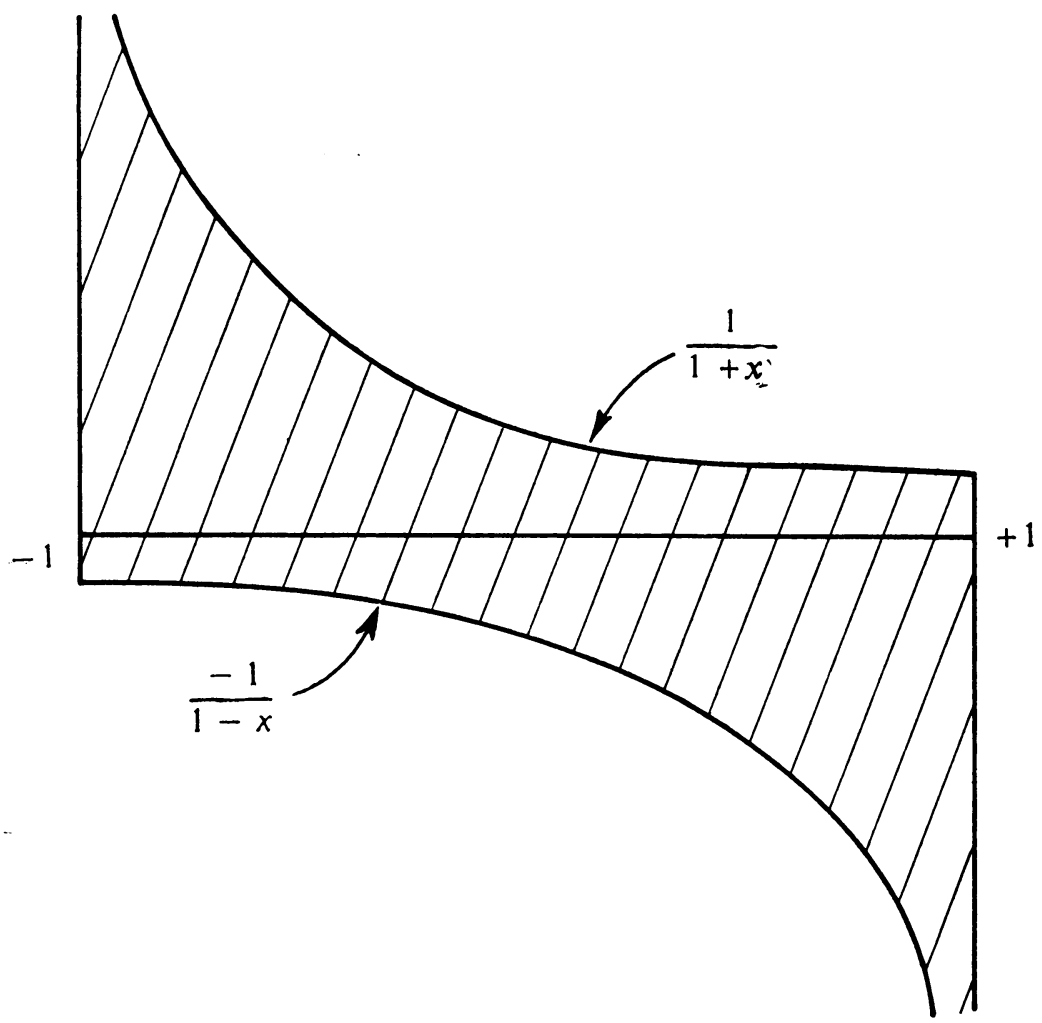

FIGURE 1. The range of $F(A, x)=a /(1+a x)$

THEOREM 2. If Statement B is valid for every continuous function then Statement $\mathrm{C}$ is valid for every continuous function.

Thus Haar's problem $[1 ; 2]$ which is the study of the implications of Statement C is subsumed by the study of the implications of Statement B.

\section{REFERENCES}

1. N. I. Achieser, Theory of approximation, Ungar, New York, 1956.

2. A. Haar, Die Minkowskische Geometrie und die Annäherung an stetige Funktionen, Math. Ann. 78 (1918), 244-311.

3. T. S. Motzkin, Approximation by curves of a unisolvent family, Bull. Amer. Math. Soc. 55 (1949), 789-793.

4. J. R. Rice, Criteria for the existence and equioscillation of best Tchebycheff approximations, J. Res. Nat. Bur. Standards. Sect. B 64 (1960), 91-93.

5. - The characterization of best nonlinear Tchebycheff approximations, Trans. Amer. Math. Soc. 96 (1960), 322-340.

6. C. Goffman, Real functions, Rinehart, New York, 1953.

General Motors Research Laboratories, WARREN, MICHIGAN 